\title{
Cylindrical Liquid Crystal Lenses System for Autostereoscopic 2D/3D Display
}

\author{
Chih-Wei Chen*, Yi-Pai Huang, Yu-Cheng Chang, Po-Hao Wang, Po-Chuan Chen \\ Department of Photonics \& Institute of Electro-Optical Engineering, Display Institute, \\ National Chiao Tung University, Hsinchu, Taiwan, 30010 \\ 1001 Ta Hsueh Road, CPT Building, Room 502, Hsinchu, Taiwan, R.O.C 30010 \\ Chao-Hsu Tsai
Electronics and Optoelectronics Research Laboratories of the Industrial Technology Research Institute (EOL/ITRI), Hsinchu, Taiwan, R.O.C 31040

\begin{abstract}
The liquid crystal lenses system, which could be electrically controlled easily for autostereoscopic 2D/3D switchable display was proposed. The High-Resistance liquid crystal (HRLC) lens utilized less controlled electrodes and coated a high-resistance layer between the controlled-electrodes was proposed and was used in this paper. Compare with the traditional LC lens, the HR-LC Lens could provide smooth electric-potential distribution within the LC layer under driving status. Hence, the proposed HR-LC Lens had less circuit complexity, low driving voltage, and good optical performance also could be obtained. In addition, combining with the proposed driving method called dual-directional overdriving method, the above method could reduce the switching time by applying large voltage onto cell. Consequently, the total switching time could be further reduced to around 2second. It is believed that the LC lens system has high potential in the future.
\end{abstract}

Keywords: 2D/3D switchable, Liquid crystal lens, High-resistance layer, Autostereroscopic display.

\section{INTRODUCTION}

The liquid crystal (LC) ${ }^{1-4}$ lens are one of the most favorable designs for the 2D/3D switching display applications ${ }^{5-8}$. The purpose are that the device let viewers' right and left eyes perceived different images from the display through the concept of binocular disparity ${ }^{9}$. Since the degradation of image quality as showing $2 \mathrm{D}$ image by using the previous fixed barrier or lenticular lens type display ${ }^{10,11}$., thus, 2D/3D switchable should be necessary. When applying certain voltages, the local electric fields are formed, and then the non-uniformed electric field distribution causes the liquid crystal material reorient that the refractive index changes accordingly. Thus, the phase difference is achieved and results in the change of light direction The High-Resistance Liquid Crystal (HRLC) lens, coating a high-resistance layer into the slits between each electrode as shown in Fig.1, is proposed not only for solving this issue, but also for improving such as high crosstalk and worse focusing ability of traditional LC lens. The high-resistance layer can let the leakage current pass through resistance layer when applying difference voltages on the electrodes, and then different voltage distributions can be produced on the resistance layer simultaneously. Therefore, good focusing ability, low operating voltage, and low crosstalk LC lens will be obtained. The focusing time is another issue for the liquid crystal lens. The LC response time is too slow to be switch between on and off because of the bulk of the LC cell gap. Even if the bulk LC lens can be operate by overdrive method ${ }^{12}$ to reduce the time from initial stateto focusing state $\left(\mathrm{t}_{\text {lens-on }}\right)$, but the time from focusing to initial state ( $\left.\mathrm{t}_{\text {lens-off }}\right)$ still cannot be reduced. According to this requirement, The Dual directional overdrive method ${ }^{13}$ which can provide dual directional electro-field is proposed as well. Therefore, the response time ( $\mathrm{t}_{\text {lens-on }}$ and $\left.\mathrm{t}_{\text {lens-off }}\right)$ can be further reduced successfully through the proposed method.

\section{PRINCIPLE AND SIMULATION}

To get the better focusing ability of liquid crystal lens, the refractive index distribution in the LC layer played an important role. From the formula of GRIN lens ${ }^{14}$, the effective refractive index ( $\left.\mathrm{n}_{\text {eff }}\right)$ distribution must consist with the parabolic curve which derived in (1).

*tedd0202.di96g@g2.nctu.edu.tw; phone+886 3 571-2121 ext 59210; fax +886 3 573-7681

Three-Dimensional Imaging, Visualization, and Display 2012, edited by Bahram Javidi,

Jung-Young Son, Proceedings of SPIE Vol. 8384, 83840W · ( 2012 SPIE

CCC code: $0277-786 \mathrm{X} / 12 / \$ 18 \cdot$ doi: $10.1117 / 12.920266$ 
Here $\Delta \mathrm{n}$ is ne (extraordinary) - no (ordinary), $\mathrm{d}$ is the cell gap, $\mathrm{r}$ is the lens radius, and $\mathrm{f}$ is the focal length.

$$
\Delta n \times d=\frac{r^{2}}{2 f}
$$

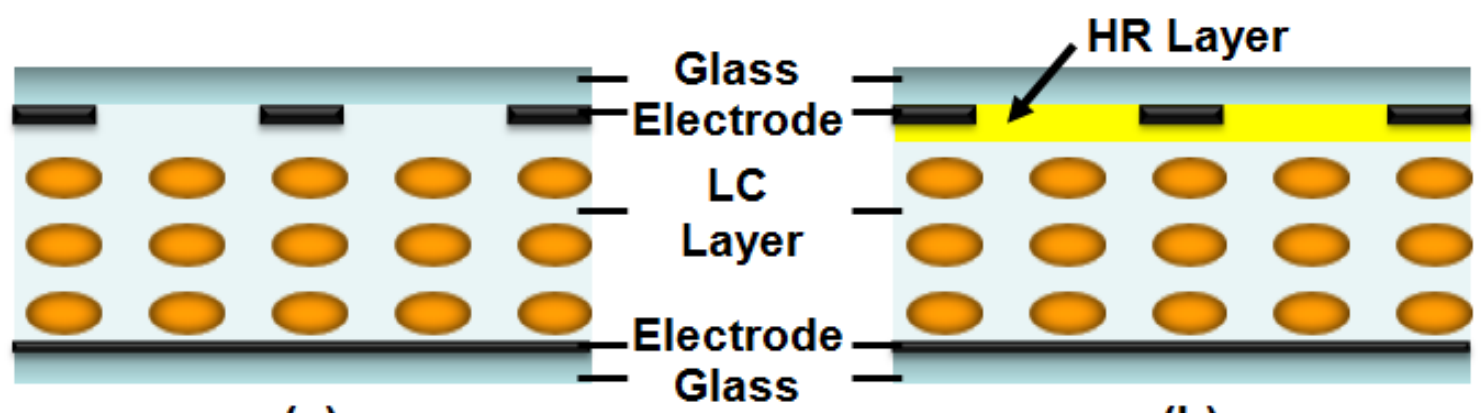

(a)

(b)

Figure 1. Sketch of (a) traditional LC lens and (b) the proposed High-Resistance LC lens.

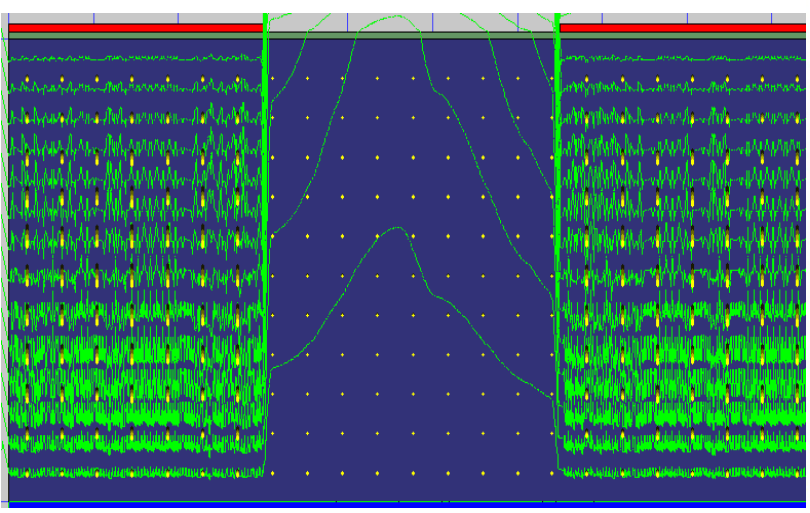

(a)

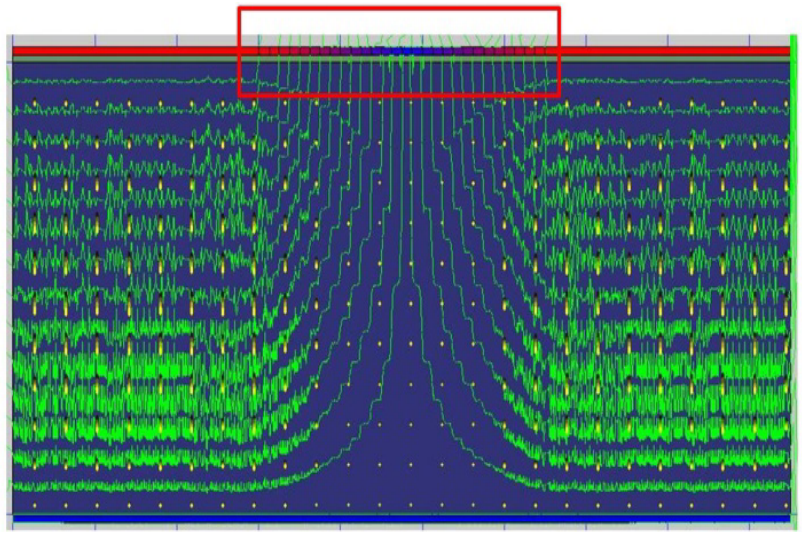

(b)

Figure 2. Illustration of the electric field distribution of (a) traditional LC lens (b) High-Resistance LC lens in simulating software.

The LC director profile and electric field distribution were calculated by software 2D-MOS or ExpertLCD as shown in Fig.2. For the traditional LC lens, the large slit within electrode make the discrete electric filed distribution as shown in Fig. 2(a); on the contrary, for the HRLC lens and traditional LC lens as shown in Fig. 2(b), due to the high resistance layer shared the electric field, thus the smooth electric filed was formed. It also meant the LC under the slit could be affected thus a gradient refractive index distribution was obtained and was closer to the ideal parabolic curve than the traditional one.

In the previous work, a new LC lens with superior well focusing property had generated. However, its response time was too slow because of the bulk LC cell gap. Thus, according to the formula of response time as shown in Eq. (2) ${ }^{16}$, the thin LC cell gap, fast response LC material, and high driving voltage could be used to shorter LC focusing time.

$$
t_{\text {focusing }}=\frac{\gamma_{1} d^{2} / k \pi^{2}}{\left(V / V_{t h}\right)^{2}-1}
$$

Although the overdrive method could be used to reduce the lens-on time from initial state to focusing state for the conventional method, the lens-off time switching from focusing state to initial state still could not be reduced due to the LC molecular recovered to initial state through weak anchoring force without extra external forces. Therefore, the driving method which called dual directional overdriving method was proposed as shown in Fig. 3. The LC structure was fabricated with finger-electrode on both top and bottom substrate. The Liquid crystal lens could operate at switch-on state when applying the corresponding voltages to top electrodes and reference voltages to bottom electrodes to build a 
lens-like liquid crystal distribution. And it could also apply the operating voltage as well as reference voltage alternately to both top and bottom side simultaneously to produce lateral electric field along with the initial LC alignment. Hence the LC molecules tended to aligned along the initial rubbing direction. By the dual directional electric-fields, the response time was supposed to be reduced under the extra forces.

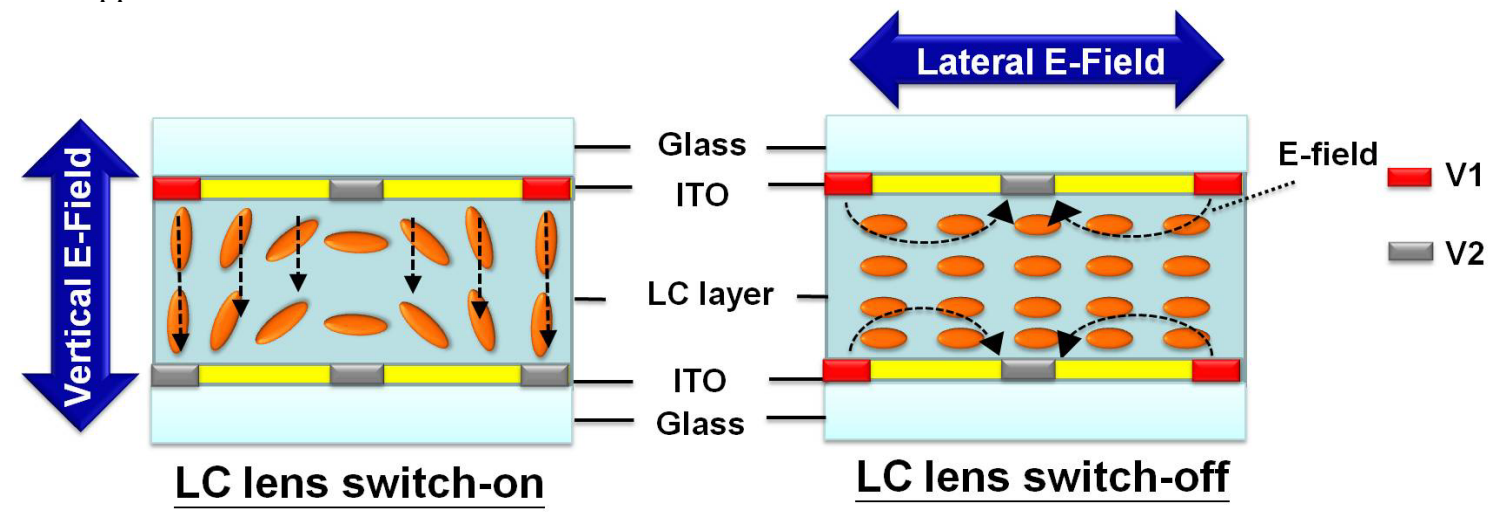

Figure 3. Illustration of Dual directional overdrive method for (a) focusing state and for (b) recovering state when applying the external lateral electric field

\section{EXPERIMENTAL RESULT}

The HRLC lens for the 3 inch LCD panel was fabricated and was measured. Its high-resistance layer with its sheet resistance was about $3 \mathrm{M} \Omega, \mathrm{E} 7$ (Merck) was used as the LC material, and the cell gap, lens pitch, and electrode width of HR LC lens were $60 \mathrm{um}, 188.5 \mathrm{um}$, and $14.5 \mathrm{um}$ respectively.

In order to affect the LC effectively, the traditional LC lens needed to provide higher operating voltage ( $\sim$ 10volt $)$ to affect the LC molecular under the slit area although it still could not affect the LC under the slit area well and matched with the ideal refractive index distribution; on the contrary, for the HR LC lens, because high-resistance layer could let the leakage current pass through it, the electric field could distribute to the slit area and then could affect the LC directly. Moreover, because the high resistance layer could conserve the electric field within it, the less power losing let the HR LC lens only needed lower voltage ( 5volt). Therefore, the benefit of low operating voltage was obtained as well.

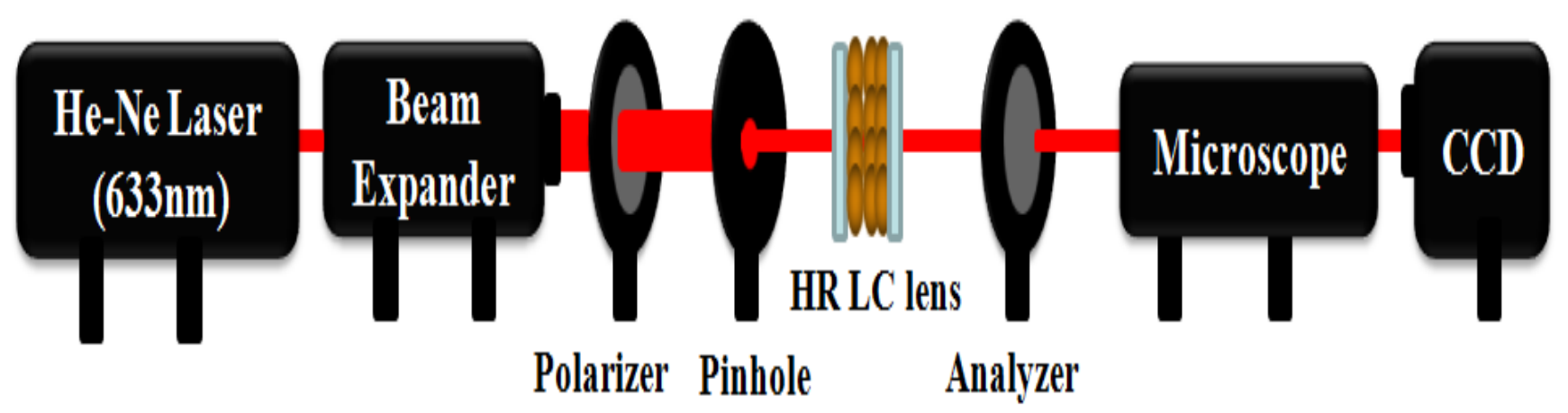

Figure 4. Experimental setup for measuring retardation of HR LC Lens

The experimental system was shown in Fig.4. He-Ne Laser is used to be the incident light for measuring the interference pattern of the LC cell. After incident light pass through the LC lens, the CCD could capture the fringe pattern to reconstruct effective refractive index $\left(\mathrm{n}_{\text {eff }}\right)$ distribution by the phase difference formula (3).

$$
\Delta \Phi=\frac{2 \pi}{\lambda} \Delta n \bullet d
$$




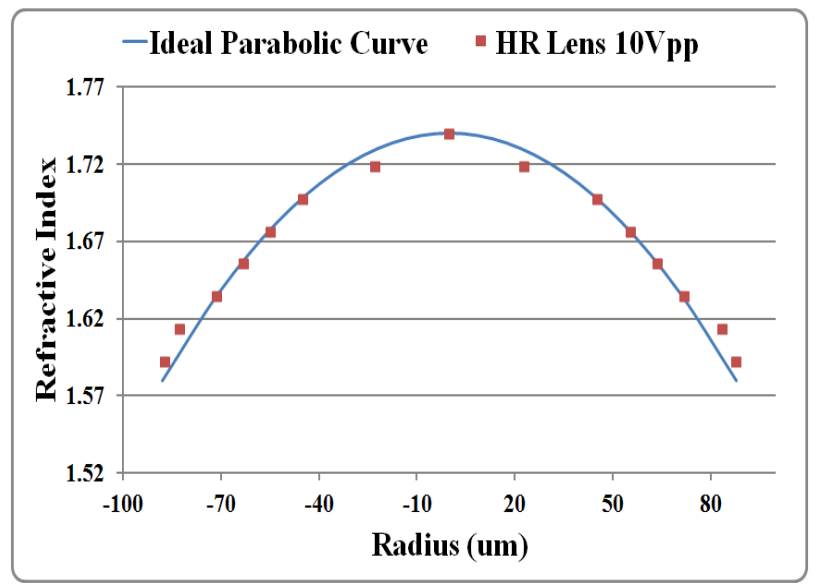

Figure 5. Effective refractive index distribution of HR LC lens under driving 5Vrms

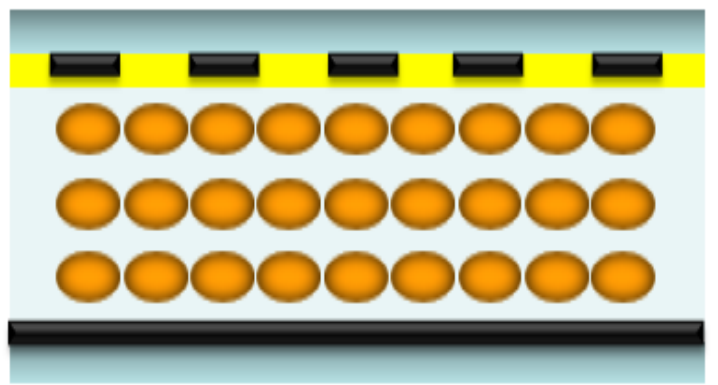

(a)

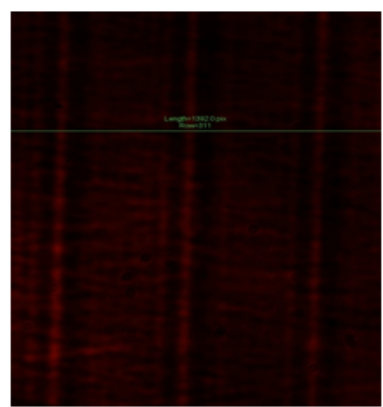

(c)

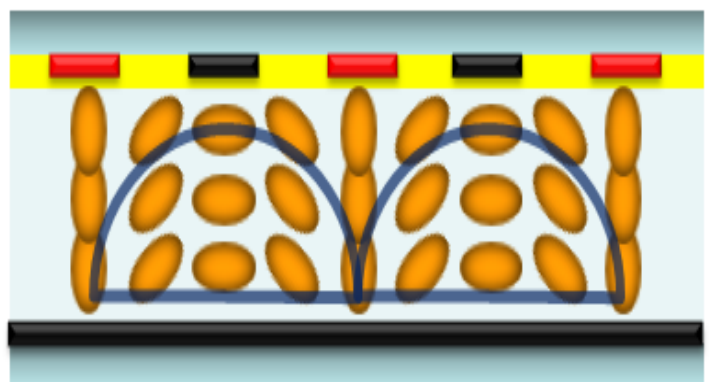

(b)

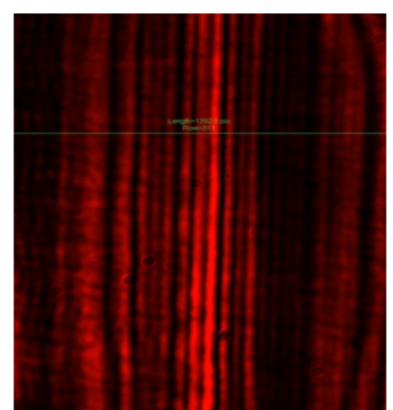

(d)

Figure 6. (a) Illustration and (c) retardation image of two HR LC Lens without driving, and (b) illustration and (d) retardation of two HR LC Lens under driving

$\Delta \Phi$ is the phase difference, $\Delta \mathrm{n}$ is ne (extraordinary) - no (ordinary), $\mathrm{d}$ is the pattern distance, and $\mathrm{k}$ is the wave number. The sketches of operating before and after focusing were shown in Figure.6 (a)\&(c). The captured fringe images of before and after focusing were also shown in Fig.6 (b)\&(d). According to the measured results, the reconstructed refractive index distribution of HR LC lens was obtained and its result was closed to the ideal parabolic curve as shown in Fig.5. Hence, it is believed that the proposed HRLC lens had the properties of good focus ability and the lower aberration for 3D image.

Combining the proposed HRLC lens with the LCD panel, the angular distribution was measured as shown in Fig. 7. The HRLC lens array here was driven under $5 \mathrm{Vrms}$ for the specific focal length. According to the measurement, the crosstalk was about $25 \%$ between left and right views. The crosstalk could be further reduced when the refractive index distribution as close as to the ideal parabolic curve. In addition, the crosstalk also could be reduced using the digital crosstalk reduction method ${ }^{18}$ that the crosstalk could be reduced as well through the image processing. Finally, a captured image from the 3 inch 2D/3D switchable autostereoscopic display by HRLC lens was shown in Fig.8. 


\section{Angular Distribution}

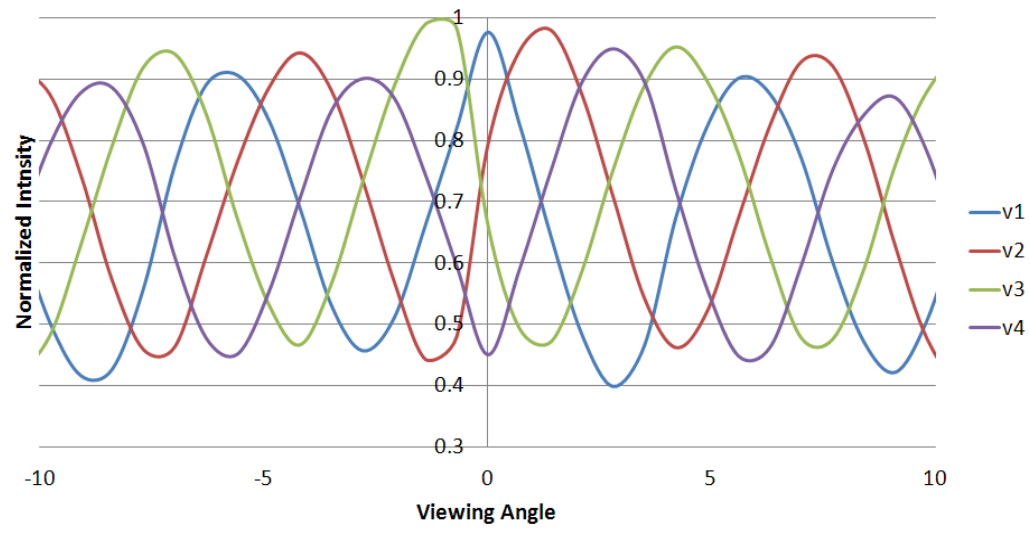

Figure 7. Angular distribution of GDLC

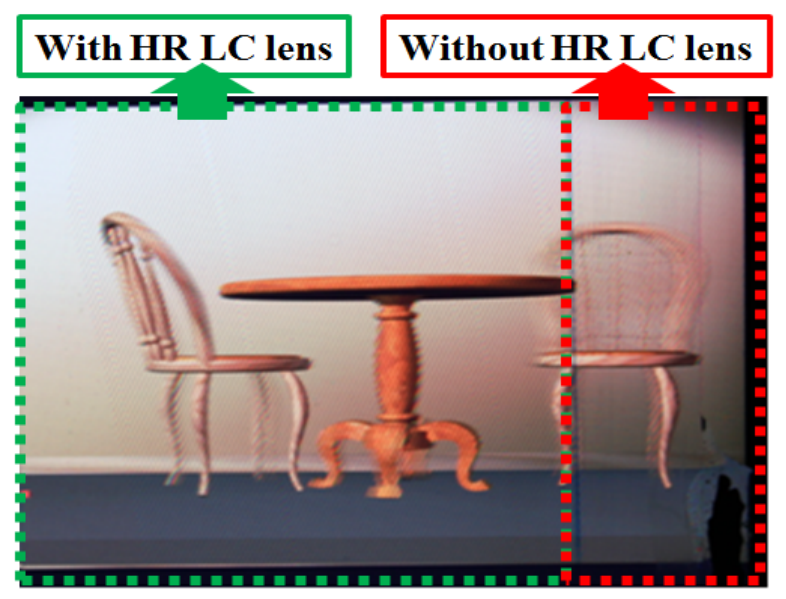

Figure 8. 3D Images with / without using HR LC lens.

The result could be categorized to two parts: the lens-on state and the lens-off state. The lens-on time of LC lens took about 6 seconds from initial state to focusing state without the overdrive method as introduced above. By applying the overdrive method, the focusing time could be reduced to 1.0 seconds. Similarly, for the lens-off time, it took about 4 seconds to regain without applying lateral electric field. By using the proposed operating method to produce lateral electric field, the lens-off time could be decreased to 1.4 seconds as well. Moreover, the low operating voltage was the other benefit. Only $2.5 \mathrm{~V}_{\text {rms }}$ was needed to accelerate the lens-off time. The sequential images during focusing and recovering states were captured and were also shown in Fig.9. From the result, the much faster response LC lens was achieved and demonstrated. Furthermore, the overall response time ( $t_{\text {lens-on }}$ and $\left.t_{\text {lens-off }}\right)$ of the proposed method was reduced by $75 \%$ comparing with the conventional LC lens.

\section{CONCLUSION}

The High-Resistance Liquid Crystal (HRLC) lens for the 2D/3D switching display with high focusing ability and low crosstalk had been proposed. The HRLC lens provided a voltage distribution that is closer to the parabolic curve than the traditional LC lens. Besides, because the high-resistance layer let the leakage current pass through resistance layer, the HRLC lens just needed the voltage that is lower than the traditional LC lens's driving voltage but still performed the same focus length. However, the switching time was still a issue for the bulk LC cell. A specific driving method called dual-directional overdriving method was proposed as well to not only reduce the lens-on time, but also reduce the lensoff time. This method could reduce the switching time by applying large voltage onto cell. Consequently, the switching time ( $t_{\text {lens-on }}$ and $t_{\text {lens-off }}$ ) could be further reduced to $\sim 2$ seconds (general it took 10 seconds). 


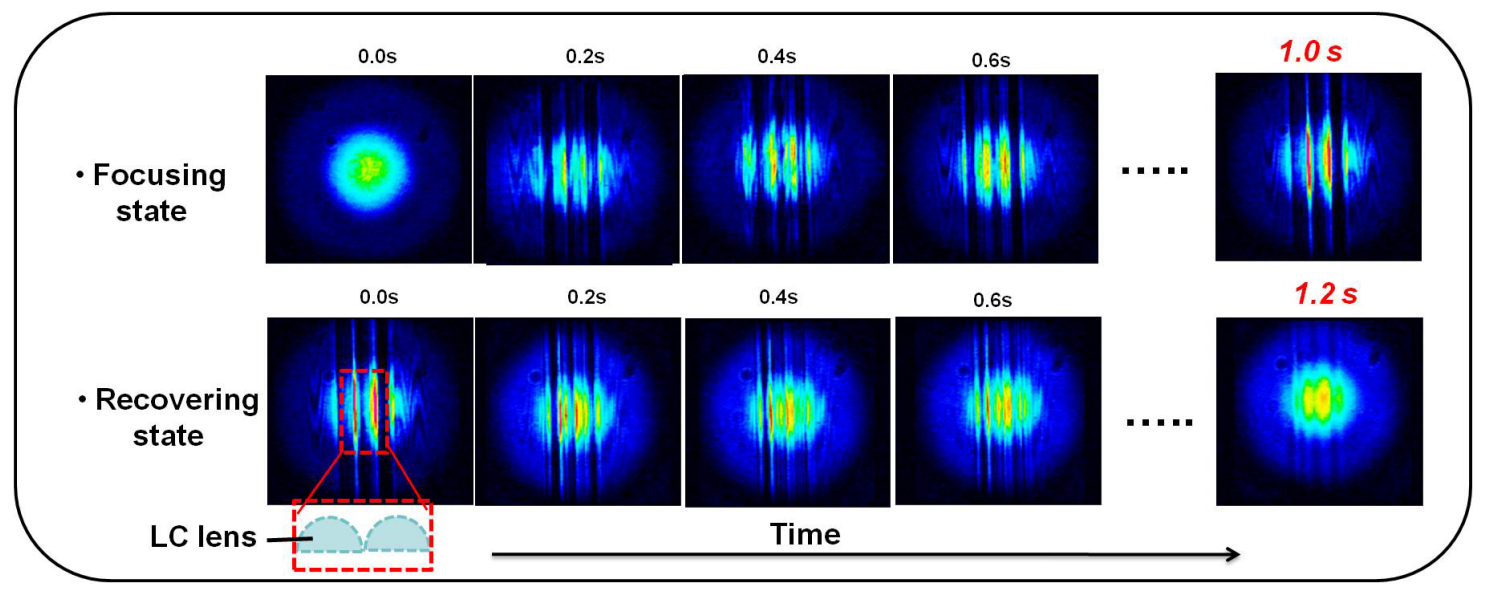

Figure 9. Light distribution of proposed LC lens under focusing

\section{ACKNOWLEDGEMENT}

This work was partially supported by National Science Council, and ITRI, Taiwan, for Academic Projects No.NSC992220-E-009-006. We also would like to thanks Mrs. S. F. Lin, Mr. J. H. Wu, and Mr. L. Hsu for their technical support. And we also would lie to thanks Mr. J. J. Li in SuperD company for his advices.

\section{REFERENCES}

[1] S. Sato, "Liquid-crystal lens-cells with variable focal length," Jpn. J. Appl. Phys.18, pp. 1679-1684 (1979)

[2] H. W. Ren, Y. H. Fan, S. Gauza, and S.T. Wu, "Tunable-Focus Cylindrical Liquid Crystal Lens," Jpn. J. Appl. Phys.43, pp. 652-653 (2004).

[3] H. W. Ren, D. W. Fox, B. Wu, and S. T. Wu, "Liquid crystal lens with large focal length tunability and low operating voltage," Opt. Express 15, pp. 11328-11335 (2007).

[4] A.F. Naumov, M. Y. Loktev, I. R. Guralnik, and G. Vdovin, "Liquid-Crystal adaptive lenses with modal control," Optics Letters 23, pp. 992-994(1998)

[5] Y. P. Huang, L. Y. Liao, and C. W. Chen, "2D/3D switchable autostereoscopic display with multi-electrically driven liquid crystal lenses," J. Soc. Info. Display 18, pp.642- 646 (2010)

[6] C. W. Chen, Y. C. Huang, Y. P. Huang, and J. F. Huang, "Fast Switching Fresnel Liquid Crystal Lens for Autostereoscopic 2D/3D Display", SID Symposium Digest 41, pp. 425-428 (2010)

[7] H. K. Hong, S. M. Jung, B. J. Lee, and H. H. Shin, "Electric-Field-driven LC lens for 3-D/2-D autostereoscopic display," J. Soc. Info. Display 17, pp.399- 406, (2009).

[8] J. Flack, J. Harrold, and G. J. Woodgate, "A prototype 3-D mobile phone equipped with a next generation autostereoscopic display,” Proc. SPIE 6490, pp. 1-12 (2007)

[9] B Javidi and F. Okano, [Three-Dimensional Television, Video, and Display Technologies], Springer, pp.461 (2002).

[10] G. J. Woodgate, J. Harrold, A. M. S. Jacobs, R.R. Moseley, and D. Ezra, "Flat-panel autostereoscopic displays: characterization and enhancement," Proc. SPIE 3957, pp. 153-164 (2000).

[11] C. V. Berkel and J. A. Clarke, "Characterization and optimization of 3D-LCD module design," Proc. SPIE 3012, pp. 179-187 (1997).

[12] L. Y. Liao, P. Y., Shieh, and Y. P. Huang, "Marginal electrodes with over-drive method for fast response liquid crystal lens applications," SID Symposium Digest 41, pp. 1766-1769, (2010).

[13] P. C. Chen, C. W. Chen, Y. P. Huang, and J. J. Li, "Dual directional Overdriving Method for Fast Response LClenses on Autostereoscopic 3D Display", SID Symposium Digest 42, pp.17-20, (2011).

[14] E. Hecht, [Optics], Addison Wesley, (2002).

[15] J. W. Goodman, [Introduction to Fourier Optics], McGraw-Hill, (1968)

[16] D. K. Yang and S. T. Wu,[ Fundamentals of Liquid Crystal Devices], John Wiley \& Sons, (2006).

[17] S. T. Wu and D. K. Yang, [Reflective Liquid Crystal Displays], Wiley, pp.270-271, (2001)

[18] Y. C. Chang, C. Y. Ma, and Y. P. Huang, "Crosstalk Suppression by Image Processing in 3D Display," SID Symposium Digest 42, pp. 124-127 (2011) 